\title{
Quantitative demonstration of the superiority of circularly polarized light in fog environments
}

\author{
Sara Peña-Gutiérrez, ${ }^{1, *}$ (i) Maria Ballesta-Garcia, ${ }^{1}$ (i) Pablo García-Gómez, ${ }^{1,2}$ and \\ SANTIAGo ROYO ${ }^{1}$ \\ ${ }^{1}$ Centre de Desenvolupament de Sensors, Instrumentació i Sistemes, Universitat Politècnica de Catalunya (UPC-CD6), Rambla Sant Nebridi \\ 10, E08222, Terrassa, Spain \\ ${ }^{2}$ Beamagine S.L., C/Bellesguard 16, E08755, Castellbisbal, Spain \\ ${ }^{*}$ Corresponding author: sara.pena.gutierrez@upc.edu
}

Received 7 October 2021; accepted 16 November 2021; posted 29 November 2021; published 5 January 2022

\begin{abstract}
The polarization behavior of light transmitted through scattering media is studied quantitatively. A division of focal plane (DOFP) imaging polarimeter modified with a wideband quarter-wave plate (QWP) is used to evaluate the linear and circular depolarization signals. This system allows the measurement of the linear and circular co-polarization and cross-polarization channels simultaneously. The experiments are carried out at CEREMA's $30 \mathrm{~m}$ fog chamber under controlled fog density conditions. The polarization memory effect with circularly polarized light is demonstrated to be superior in forward transmission compared to the same phenomena with linearly polarized light when imaging inside a scattering medium. This paves the way for its use in imaging through scattering media for hazard detection in different applications.
\end{abstract}

(C) 2022 Optica Publishing Group under the terms of the Optica Open Access Publishing Agreement

https://doi.org/10.1364/OL.445339

Vision through turbid media is an important issue that comes into play when working in fields as diverse as medical diagnosis [1], remote sensing, transport, and surveillance [2]. Specifically, imaging through scattering media such as fog, haze, or smoke is of special interest in highly up-to-date applications such as autonomous vehicles or surveillance tasks, whether in terrestrial, maritime, or aerial environments.

In such turbid media, there are two important types of photons: ballistic and diffuse photons, which depend on the number of scattering events they undergo along their path. Ballistic photons are those unaffected by the presence of the turbid media, while diffuse photons take randomly larger paths due to multiple scattering events, so the laws of geometrical optics are not enough to describe them [3]. Different approaches to detecting ballistic photons through turbid media have been studied with the aim of enhancing sensor detectivity in such environments. Theoretical simulations have analyzed the behavior of polarized light in forward scattering, and despite ideal conditions, the main conclusion was that the behavior depends on the input polarization state [4-6]. Other authors have experimentally proven the results given by simulations; specifically, that the depolarization of the beam during the propagation is proportional to the optical depth, although this result is restricted to linear polarization [7]. In this paper, we propose to analyze the properties of polarized light to demonstrate quantitatively its advantage compared to conventional intensity detection schemes in turbid media [8,9]. The benefit to be presented comes from the so-called polarization memory effect, which has been broadly studied $[10,11]$. However, to the best of our knowledge, nobody has experimentally proven its application at long distances under real fog conditions by comparing the effectivenesses of circular and linear polarization imaging.

To study the behavior of linear and circular polarization, polarization imaging is required. Hence, one should measure the Stokes vector $\vec{S}$ by taking different intensity measurements [1]. As a polarization detector, we used a Phoenix 5.0 MP color polarization camera (LUCID Vision Labs) together with an objective of fixed focal length (EO \#59-871) embedded within an aluminum IP68 housing case for water protection. This camera is a division of focal plane (DOFP) imaging polarimeter that allows the intensity from the parameter $S_{0}$ and the linear Stokes components of light $\left(S_{1}\right.$ and $\left.S_{2}\right)$ to be measured. The camera incorporates the Sony IMX250MYR CMOS sensor, which consists of an array of micropolarizers oriented at four different angles. To measure the polarization, a polarimetric measurement basis must be defined. For this purpose, we define a macro pixel formed by four pixels corresponding to the angles of linear polarization $0,45,90$, and $135^{\circ}$, as shown in Fig. 1(a). The instrument matrix $A$ of this macro pixel contains the transformation carried out by this macro pixel to convert the input polarization to intensity information detected by the DOFP camera at each channel, as shown below:

$$
I=A \cdot \vec{S}
$$

where $I$ denotes the intensity, $\vec{S}$ is the input Stokes vector, and $A$ is the instrument matrix.

The instrument matrix rows are calculated by extracting the first row of the Mueller matrix of each state from the measurement basis. In this case, four simultaneous measurements are performed using the four different micropolarizers $(0,45,90$, and $135^{\circ}$ ). Hence, the instrument matrix $A_{L}$ (from linear 


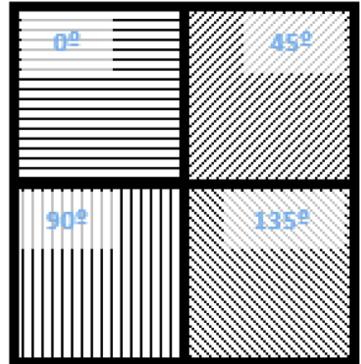

(a)

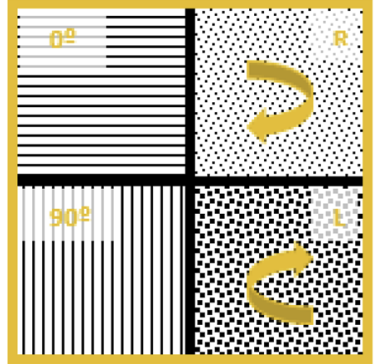

(b)
Fig. 1. (a) Scheme of a DOFP camera macro pixel comprising four micropolarizers whose optical axes are oriented at 0, 45, 90, and $135^{\circ}$, respectively. (b) Modification of the measuring states when the QWP (gray/yellow border) is placed in front of the DOFP camera. The channels corresponding to the angles 45 and $135^{\circ}$ are expanded to detect left-handed and right-handed circular polarization, respectively. The linear channels 0 and $90^{\circ}$ remain unchanged.

configuration) is:

$$
A_{L}=\frac{1}{2}\left(\begin{array}{cccc}
1 & 1 & 0 & 0 \\
1 & 0 & 1 & 0 \\
1 & -1 & 0 & 0 \\
1 & 0 & -1 & 0
\end{array}\right) .
$$

This matrix $A_{L}$ allows the detection of the linear polarization states $\left(S_{0}, S_{1}, S_{2}, 0\right)$. Since our goal was to simultaneously measure both the linear and the circular polarization states and compare their behavior, an achromatic quarter-wave plate (QWP; Thorlabs, AQWP10M-580) was placed in front of the objective and aligned with the optical axis of the micropolarizer at $0^{\circ}$ to measure the parameter $S_{3}$. In this case, the DOFP camera is no longer a linear Stokes detector but a snapshot orthogonal state contrast imager since we are no longer able to detect the parameter $\mathrm{S}_{2}$. This modification can be expressed theoretically by considering the Mueller matrix of the QWP when calculating the instrument matrix. Then, the new instrument matrix $A_{C}$ (from circular configuration) of the polarization state analyzer (PSA) is

$$
A_{C}=\frac{1}{2}\left(\begin{array}{cccc}
1 & 1 & 0 & 0 \\
1 & 0 & 0 & 1 \\
1 & -1 & 0 & 0 \\
1 & 0 & 0 & -1
\end{array}\right) .
$$

Considering this, if we look at the new $A_{C}$, the former $45^{\circ}$ and $135^{\circ}$ channels can now discriminate left-handed (L) and right-handed $(\mathrm{R})$ circular polarization states instead of detecting linearly polarized light (as shown in Fig. 1). Since the QWP's fast axis is parallel to the $0^{\circ}$ linear polarizer, the horizontally $\left(0^{\circ}\right)$ and vertically $\left(90^{\circ}\right)$ linearly polarized light remain unaltered as they are eigenvalues of the retarder's Mueller matrix. These changes are explained in Fig. 1(b).

After this modification, the camera was calibrated to get the correct alignment between the camera and the QWP. The procedure consisted of measuring the fast axis of the QWP using a polarimeter (Thorlabs, PAX1000VIS); then the QWP axis was aligned with respect to that of the micropolarizer at $0^{\circ}$. The change in the aperture of the objective did not affect the polarization state, showing that the QWP axis was properly aligned with that of the camera.
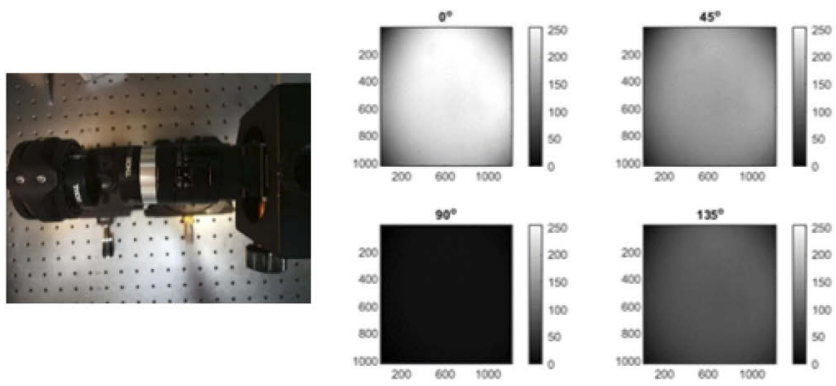

Fig. 2. Setup of the DOFP camera together with the QWP aligned with the horizontal axis (left) and the intensity of the polarized channels that detect the input horizontal polarization (right).

In order to assure the correct position of the micropolarizers, horizontally polarized light was measured using this setup. The horizontal $\left(0^{\circ}\right)$ channel detected the maximum signal, as shown in Fig. 2. Afterwards, a radiometric calibration was performed to tackle non-uniform responses due to the variance in pixel detectivity and the dependence of the transmission of the micropolarizers on their orientation. The latter issue can be appreciated by examining the $45^{\circ}$ and $135^{\circ}$ channels in Fig. 2, where the intensity values differ even though they are expected to be equal.

The experiments were conducted at CEREMA's large-scale fog chamber located in Clermont-Ferrand (France) [12]. This can produce homogeneous fog under controlled conditions in controlled steps of desired optical visibility. In this case study, a cycle of fog with visibilities ranging from 5 to $40 \mathrm{~m}$ was measured. The visibility was monitored by measuring the transmitted power when using a continuous laser, as explained in [13]. The particle size distribution of the water droplets corresponded to a Gaussian distribution with a mean diameter of $2 \mu \mathrm{m}$ : very similar to the statistics of natural radiation fog [14]. During the test, active polarized illumination was used. In the illumination system, white light was passed into a polarization state generator (PSG) with the proper polarizer for each case. The test consisted of illuminating the chamber in transmission geometry with two different polarization states: horizontal linear polarization and left-circular polarization. The forward-scattered light properties were analyzed using the images acquired by the modified DOFP camera.

During the experiments, the fog chamber was completely empty of objects, so there were no undesired interactions with them within the field of view (FOV) of the DOFP camera. Figure 3 shows the final setup of the DOFP camera, including the QWP and the IP68 cage. The camera was set to $1 \mathrm{~s}$ of exposure time and no gain, and the luminous flux emitted under circularly and linearly polarized illumination was adjusted to be the
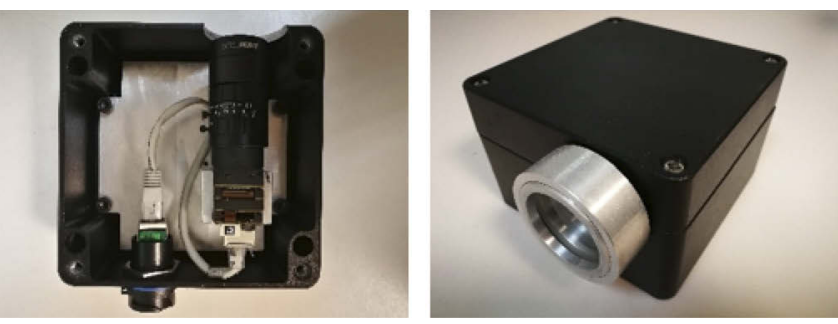

Fig. 3. Setup of the DOFP camera together with the QWP aligned with the horizontal axis (left) and enclosed camera within an IP68 cage to preserve the seal of the inner cavity (right). 


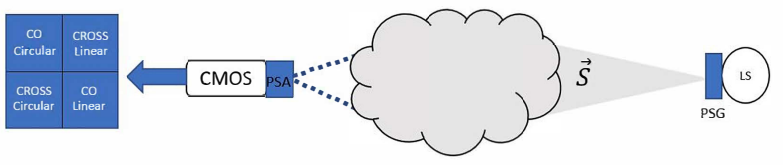

Fig. 4. Scheme of the experiment where active polarized illumination radiates through the fog and reaches the DOFP camera. LS, light source; $\vec{S}$, input polarization state.

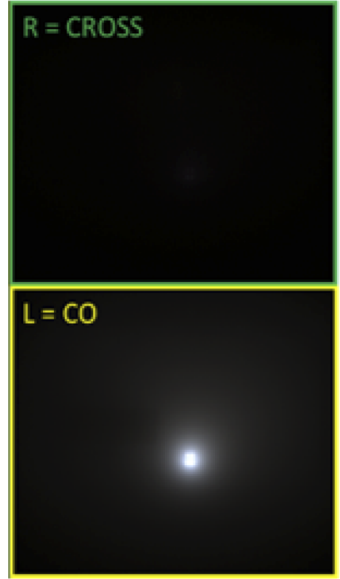

(a)

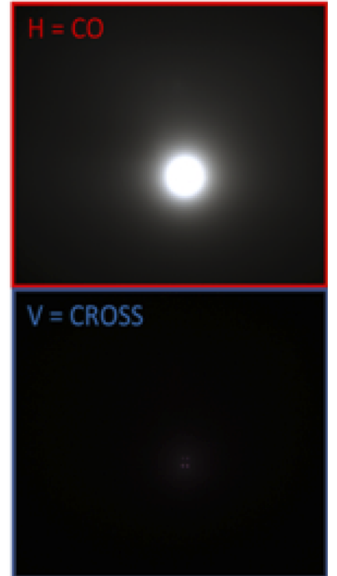

(b)
Fig. 5. Intensity detected in the $\mathrm{CO}$ and CROSS channels with very dense fog (visibility $<10 \mathrm{~m}$ ) when the input illumination is $\mathrm{L}$ polarized (a) and $\mathrm{H}$ polarized (b). The CROSS channels filter the active illumination and the $\mathrm{CO}$ channels probe the prevalence of the input polarized light through the fog.

same in both repetitions during the whole cycle. The light source had a divergence of $\pm 6.65^{\circ}$ and was aligned pointing towards the camera, which was located at a distance of $28 \mathrm{~m}$. Since the polarization could be adjusted to be horizontal linear and left-handed circular polarization, we defined the channels of the detector as follows: the co-polarized (CO) channel was the channel matching the active illumination, and the cross-polarized (CROSS) channel was for orthogonally polarized active illumination. A scheme of the setup is presented in Fig. 4.

It may be observed in Fig. 5 that the $\mathrm{CO}$ channels present a circle of saturation at the position of the light source for both input polarization states (left-circular: L, linear horizontal: H). Initially, we aimed to study the depth to which polarized light could penetrate through the fog and reach the detector as a function of the visibility for both linear and circular polarization. However, the light source was visible even for low visibilities with the lowest optical power at the furthest possible distance at normal illumination to the camera. Only under extremely dense fog (visibility of less than $5 \mathrm{~m}$ ) were all channels completely dark. Despite this, as the visibility increased, so did the dominance of detected CO-channel photons.

Also, we noticed that visibility affected the saturation radii on the CO images obtained. The denser the fog, the shorter the radius of the saturation circle in the image. This effect occurs because light undergoes multiple scattering events locally at denser fog levels, getting more diffused and depolarized. Quantitative measurements of the size of the saturation veil are shown in Fig. 6. Only the ballistic photons maintaining the polarization

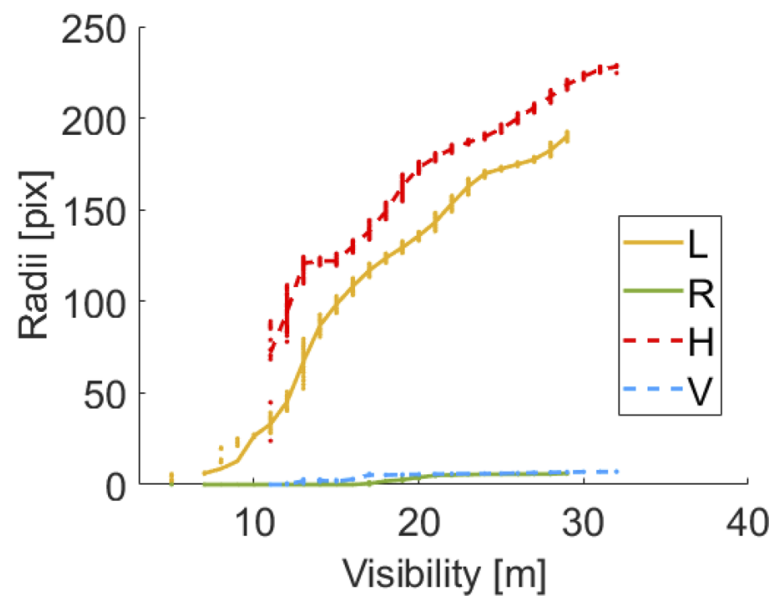

Fig. 6. Comparison of the saturation veil radii in pixels for the $\mathrm{CO}$ and CROSS channels for circular and linear polarization as a function of the visibility.

arrive at the detector. Thus, we aimed to compare the abilities of linear and circular polarization to maintain their initial properties based on the radius of the circle in the saturation veil [15].

The saturation radius is directly proportional to the source divergence and the visibility of the turbid media. The light source radius is reduced at low visibilities because there is a higher probability of single and multiple scattering events that randomize the photon directions, preventing them from arriving at the detector. As the fog gets lighter, the saturation beam radius starts to grow because the probability of being scattered is reduced. The radius will tend to achieve the initial width when there is no fog in the chamber.

Comparing the polarization signals, the radii in the L channel are always smaller (0.78 times smaller) than those in the $\mathrm{H}$ channel. This confirms that circular polarization can penetrate deeper in fog without depolarizing, as more ballistic photons are detected than in the case of linear polarization. This behavior is explained by the Mie scattering phase functions of linear and circular polarization. The phase function in the circular case possesses a marked forward lobule that permits the photons to propagate around the beam axis with higher probability than in the linear case [4]. In addition, the radii of the CROSS channels, $\mathrm{R}$ (right-circular) and V (linear vertical), denote the presence of multiple scattering that changes the initial polarization of the photons and the off-axis illumination from the divergence of the source.

In order to confirm the result beyond the use of the saturation radius, the evolution of the radius at 15 equally spaced, nonsaturated intensity levels in the beam profile imaged at the sensor was evaluated to improve the accuracy of the method. Three different visibilities [plotted in orange $(12 \mathrm{~m})$, green $(18 \mathrm{~m})$, blue $(24 \mathrm{~m})$ ] were included to generalize the analysis. Figure 7 shows the signal ratio of the $\mathrm{CO}$ and CROSS channels relative to the parameter $S_{0}$ plotted against the radius of the section of the intensity profile at 15 different values of intensity. Circular (solid lines) and linear (dashed lines) polarization cases are included for comparison.

The curves show that the broader the intensity profile in the CROSS channel (larger values of radius), the faster the beam is depolarized and the signal increases. It should be noted that the 

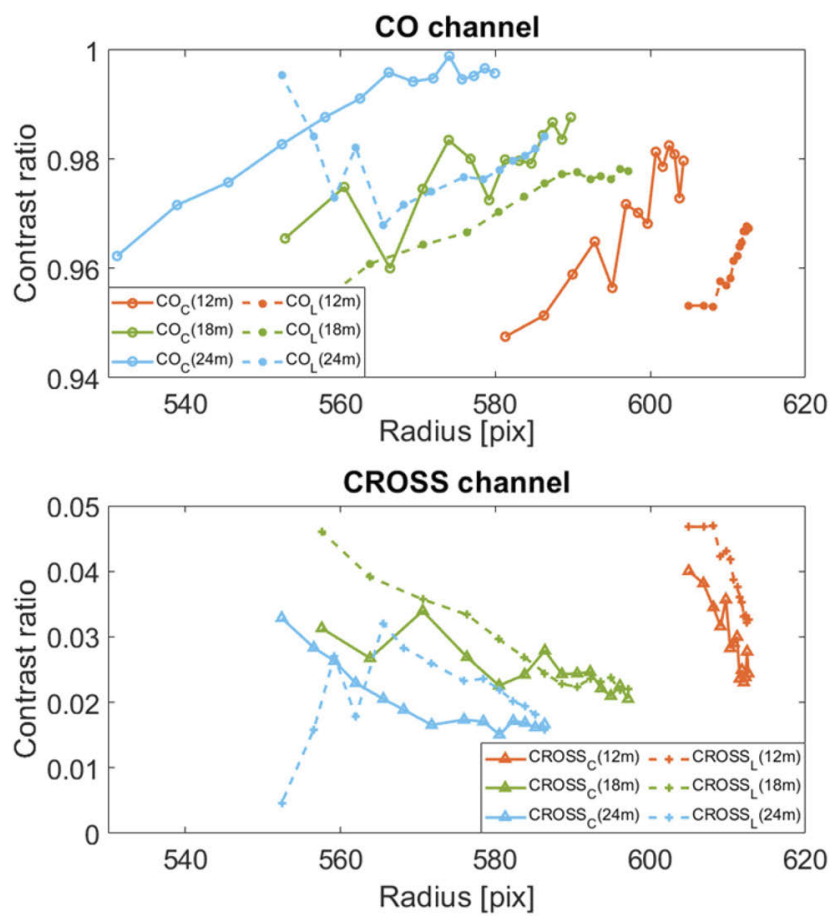

Fig. 7. Comparison of the ratio of CO (top) or CROSS (bottom) with respect to the $S_{0}$ signal as a function of the radius of the intensity profile of the source image for circular (solid lines) and linear (dashed lines) polarizations at three visibilities: 12,18 , and $24 \mathrm{~m}$.

beam broadens as the visibility is reduced, due to the increase in multiple-scattering processes. In addition, circular polarization has a higher signal in the $\mathrm{CO}$ channel and a lower one in the CROSS channel for all visibilities in comparison with linear polarization. This implies that circular polarization has a larger signal-to-noise ratio in transmission at deeper layers, whereas the signal from linearly polarized light carries some noise due to its higher depolarization ratio when propagating in scattering media.

The presented results show the dependence of the optical light penetration and the sensing range on the input polarization illumination in scattering environments. The maximum is obtained with circularly polarized light, since, at the same radius of scattering, it is detected in deeper optical layers than in the linear polarization case. On the other hand, circular polarization permits the detection of the location of the point source embedded in the scattering media with better accuracy, as it gets rid of the depolarized diffused photons. This may be useful for surveillance applications such as detecting the light from a beacon.
In conclusion, we have modified a DOFP imager into a snapshot orthogonal contrast polarization camera to simultaneously measure the Stokes linear $\left(\mathrm{S}_{1}\right)$ and circular $\left(\mathrm{S}_{3}\right)$ parameters. Using it, we have quantitatively validated and showed the differences in the behavior of linearly and circularly polarized light propagating in fog under different scattering conditions. Circular polarization was experimentally shown to exhibit the memory effect and allowed the detection of active illumination with better contrast in lower visibilities. It may be useful to test this particularity in future work with different types of scattering media such as haze and smoke.

Funding. Agència de Gestió d'Ajuts Universitaris i de Recerca (2020FI_B1 00185, 2020FI_B2 00068); European Social Fund; Ministerio de Ciencia e Innovación (MICINN) (PID2020-119484RB-I00).

Disclosures. The authors declare no conflicts of interest.

Data availability. Data underlying the results presented in this paper are not publicly available at this time as long as they were partly obtained with a privately funded project, but may be obtained from the authors upon reasonable request.

\section{REFERENCES}

1. V.V. Tuchin, J. Biomed. Opt. 21, 071114 (2016).

2. J. Scott Tyo, Dennis L. Goldstein, David B. Chenault, and Joseph A. Shaw, Appl. Opt. 45, 5453 (2006).

3. K. M. Yoo and R. R. Alfano, Opt. Lett. 15, 320 (1990).

4. J. C. Ramella-Roman, S. A. Prahl, and S.L. Jacques, Opt. Express 13, 4420 (2005)

5. T. Hu, F. Shen, K. Wang, K. Guo, X. Liu, F. Wang, Z. Peng, Y. Cui, R. Sun, Z. Ding, J. Gao, and Z. Guo, Atmosphere 10, 342 (2019).

6. J. D. van der Laan, J. B. Wright, S. A. Kemme, and D. A. Scrymgeour, Appl. Opt. 57, 5464 (2018).

7. J.S. Ryan and A.I. Carswell, J. Opt. Soc. Am. 68, 900 (1978).

8. J. Fade, S. Panigrahi, A. Carré, L. Frein, C. Hamel, F. Bretenaker, H. Ramachandran, and M. Alouini, Appl. Opt. 53, 3854 (2014).

9. E. P. Zege, A. P. Ivanov, and I. L. Katsev, Image transfer through a scattering medium, 131 (Springer-Verlag, Berlin, New York, 1991).

10. F. C. MacKintosh, J. X. Zhu, D. J. Pine, and D. A. Weitz, Phys. Rev. B 40, 9342 (1989).

11. R. Nothdurft and G. Yao, Opt. Express 14, 4656 (2006).

12. M. Colomb, H. Khaled, P. André, J. Boreux, P. Lacôte, and J. Dufour, Atmos. Res. 87, 242 (2008).

13. P. Duthon, M. Colomb, and F. Bernardin, Appl. Sci. 9, 2843 (2019).

14. P. Duthon, M. Colomb, and F. Bernardin, Atmosphere 11, 596 (2020).

15. S. Sridhar and A. Da Silva, J. Biomed. Opt. 21, 071107 (2016). 